\title{
Localization of the motor neuron somata of geniohyoid muscle in rat: A horseradish peroxidase study
}

\author{
Aimi Nadia Binti Razlan ${ }^{1}$ | Muzammil Ullah ${ }^{1}$ | Marina Yurievna Kapitonova ${ }^{2}$ | \\ Norhayati Binti Liaqat Ali Khan ${ }^{1}$ | Syed Baharom Syed Ahmad Fuad ${ }^{3}$
}

${ }^{1}$ Faculty of Dentistry, Universiti Teknologi (UiTM) MARA, Selangor, Malaysia

${ }^{2}$ Faculty of Medicine and Health Sciences, Universiti Malaysia Sarawak (UNIMAS), Sarawak, Malaysia

${ }^{3}$ Faculty of Medicine, Universiti Teknologi (UiTM) MARA, Selangor, Malaysia

\section{Correspondence}

Muzammil Ullah, Faculty of Dentistry, Universiti Teknologi (UiTM) MARA, Selangor, Malaysia.

Email: muzammilullah1940@gmail.com

\begin{abstract}
The aim of the study was to investigate the location of motor neuron somata of geniohyoid muscle in rat. Nine Sprague-Dawley rats were used in this study. Operations were performed under general anaesthesia. Nembutal sodium, 40 mg per kg intraperitoneally was used for anaesthesia. 0.02 to $0.05 \mathrm{ml}$ of $30 \%$ horseradish peroxidase (Sigma Type VI) solution in normal saline was injected into the exposed right geniohyoid muscle. After $48 \mathrm{hr}$, the animals were fixed by perfusion through left ventricle of heart, first by $100 \mathrm{ml}$ normal saline and then with $500 \mathrm{ml}$ of $1.25 \%$ glutaraldehyde and $1 \%$ paraformaldehyde in $0.1 \mathrm{M}$ phosphate buffer, $\mathrm{pH} 7.4$, at room temperature, and finally with $500 \mathrm{ml}$ of $10 \%$ sucrose in the same buffer at $4^{\circ} \mathrm{C}$. The medulla oblongata and first cervical segment of spinal cord were removed, kept in $10 \%$ sucrose in above phosphate buffer at $4^{\circ} \mathrm{C}$ for $24 \mathrm{hr}$. Thereafter, their serial transverse sections were cut in a cryostat at a thickness of $60 \mu \mathrm{m}$. The sections were treated according to tetramethyl benzidine (TMB)-horseradish peroxidase (HRP) method. HRP-labelled neuron somata were observed at the following sites: (a) In ventral part of right main hypoglossal nucleus in upper two-thirds of the closed part of medulla oblongata. (b) In ventrolateral subnucleus of hypoglossal nucleus in lower third of closed part of medulla oblongata. (c) At spinomedullary junction, they were located in dorsomedial part of right ventral grey column; a few were also seen here scattered on right side of central canal and among corticospinal fibres.

KEYWORDS

geniohyoid muscle, horseradish peroxidase, hypoglossal nucleus, motor neuron somata, rat
\end{abstract}

\section{1 | INTRODUCTION}

The geniohyoid is a muscle of neck that can move the mandible and hyoid bone during swallowing. Using retrograde degeneration technique, the geniohyoid (GH) motor neuron somata were localized in dog (Barnard, 1940), in mouse (Stuurman, 1916), and in hedgehog (Berkelbach van der Sprenkel, 1924). Using horseradish peroxidase (HRP) method, the GH motor neuron somata have been localized by Uemura et al. (1979) in cat, Krammer, Rath, and Lischka (1979) in rat, Miyazaki, Yoshida, Hirano, Shin, and Kanaseki (1981) in cat, Uemura-Sumi et al. (1981) in monkey, Yoshida, Miyazaki, Hirano, Shin, and Kanaseki (1983) in cat, Kitamura, Nishiguchi, and Sakai (1983) in rat, Kitamura, Nishiguchi, Okubo, Chen, and Sakai (1986) in rat, Uemura-Sumi, Itoh, and Mizuno (1988) in dog, rabbit and rat, Sokoloff and Deacon (1992) in monkey and Altschuler, Bao, and Miselis (1994) in rat.

There is disagreement regarding the location of the geniohyoid motor neuron somata (geniohyoid motoneurons). For example, the geniohyoid motoneurons were located in the ventrolateral subgroup of hypoglossal nucleus by Krammer et al. (1979) in rat, in the most ventral part of the ventromedial division of hypoglossal nucleus by Uemura 